\title{
Influência da função tireoidiana nos níveis séricos de CPK
}

\author{
Influence of thyroid function in CPK serum levels
}

Josivan Gomes de Lima ${ }^{1,2}$, Lúcia Helena Coelho Nóbrega ${ }^{1,2}$, Maria Lúcia Coelho Nóbrega ${ }^{2}$, Antônio Correia dos Santos Júnior', Flávia da Costa Fernandes', Deciara Jácome Torres Medeiros de Mesquita', Alexandre Barbosa Câmara de Souza'

1 Hospital Universitário Onofre Lopes (HUOL), Universidade Federal do Rio Grande do Norte (UFRN), Natal, RN, Brasil ${ }^{2}$ Centro de Endocrinologia de Natal (CEN), Natal, RN, Brasil

Correspondência para: Josivan Gomes de Lima Rua Joaquim Fabrício, 233 59012-340 - Natal, RN, Brasil josivanlima@gmail.com

Recebido em 12/Nov/2011 Aceito em 5/Mar/2012

\section{RESUMO}

Objetivo: Determinar a variação da CPK sérica em função do TSH e T4 livre (FT4) e avaliar a CPK sérica nos estados patológicos da tireoide (hipertireoidismo e hipotireoidismo), em relação ao eutireoidismo. Materiais e métodos: Analisaram-se, retrospectivamente, 6.230 exames laboratoriais de 2007 a 2011, com as dosagens deTSH e CPK. Destas, 3.369 com dosagens de FT4. Avaliou-se a correlação entre CPK com TSH e com os estados patológicos da tireoide. Resultados: A correlação entre TSH e CPK foi positiva $(r=0,065)$, enquanto entre CPK e FT4 foi inversamente proporcional $(r=-0,091, p<0,05)$. Do total, $586(9,4 \%)$ medidas foram de hipertireoidismo com mediana (intervalo) do CPK de 98 U/L (27 a 1.113) e 556 (8,9\%), hipotireóideos com CPK de 114 U/L (25-4.182). Conclusão: Houve correlação positiva entre CPK sérica e TSH e negativa com FT4, sendo a CPK menor no grupo com hipertireoidismo e maior no grupo de hipotireoidismo. Arq Bras Endocrinol Metab. 2012;56(3):190-4

\section{Descritores}

TSH; creatina fosfoquinase; T4 livre; hipertireoidismo; hipotireoidismo

\section{ABSTRACT}

Objective: To determine serum CPK variation based on TSH e freeT4 (FT4), and to assess serum CPK in pathological states of the thyroid (hyperthyroidism and hypothyroidism), in relation to the euthyroidism. Materials and methods: We evaluated retrospectively 6,230 laboratory results of TSH and CPK from 2007 to 2011. From these, 3,369 had free T4 results. We evaluated the correlation between CPK and TSH and the pathological states of the thyroid. Results: The correlation between TSH and CPK was positive $(r=0.065)$, while that between CPK and FT4 was negative $(r=-0.091, p<0.05)$. From the total of results analyzed, $586(9.4 \%)$ were measures of hyperthyroidism, with a median (range) of CPK of $98 \mathrm{U} / \mathrm{L}(27$ to 1,113), and 556 (8.9\%) were of hypothyroidism, with CPK of $114 \mathrm{U} / \mathrm{L}(25-4,182)$. Conclusion: A positive correlation was found between serum CPK and TSH, and a negative correlation between CPK and FT4. CPK was lower in the group with hyperthyroidism, and greater in that with hypothyroidism. Arq Bras Endocrinol Metab. 2012;56(3):190-4

Keywords

TSH; serum creatine kinase; free T4; hyperthyroidism; hypothyroidism

\section{INTRODUÇÃO}

A lterações musculares são frequentes em pacientes com distúrbios da tireoide, sendo um diagnóstico diferencial das miopatias. Existem vários marcadores de lesão muscular, como a lactato desidrogenase ( $\mathrm{LDH})$, a creatina fosfoquinase (CPK), aldolase (ALD), citrato sintetase (CS), aspartato aminotransferase (AST), e a alanina aminotransferase (ALT) (1).
A CPK é uma transferase que catalisa a formação da fosfocreatina a partir de ADP e creatina; essa reação armazena a energia do ATP em forma de fosfocreatina (2). No músculo a CPK está acoplada aos canais de cálcio do retículo sarcoplasmático, dependentes de ATP, relacionando-se, assim, com a atividade contrátil. Desse modo, promove a homeostase celular, gerando um circuito de alta transferência de energia a partir da fosfocreatina (3). 
A ação dos hormônios tireoidianos ao nível muscular estaria na alteração da permeabilidade da membrana plasmática pelo aumento dos canais de cálcio do retículo sarcoplasmático (4). Por isso, a disfunção tireoidiana pode causar diminuição dos canais de cálcio (hipotireoidismo) ou diminuição excessiva na fosforilação enzimática (hipertireoidismo) (5), desencadeando miopatia (6-7). Assim, 79\% dos pacientes com hipotireoidismo e $67 \%$ com hipertireoidismo acompanhados prospectivamente apresentaram alguma queixa muscular (8).

O objetivo deste trabalho é determinar como varia a CPK sérica em função dos níveis de TSH e FT4. Avaliamos também como se apresenta a CPK sérica nos pacientes com estados patológicos da tireoide (hipertireoidismo e hipotireoidismo) e suas apresentações subclínicas, em relação aos indivíduos com níveis hormonais adequados.

\section{MATERIAIS E MÉTODOS}

Foram avaliados retrospectivamente exames laboratoriais disponíveis no banco de dados de nosso laboratório e realizados em pacientes que estavam em investigação ou seguimento de alguma patologia tireoidiana no período de 2007 a 2011. As dosagens séricas $(\mathrm{N}=$ 6.230) de TSH e CPK realizadas em uma mesma coleta sanguínea foram incluídas no estudo.

O TSH foi medido pela técnica de quimiluminescência ultrassensível, de análise de sensibilidade até $0,002 \mathrm{mU} / \mathrm{L}$. Foram considerados normais os valores de 0,4 a 4,0 mU/L. As dosagens de CPK foram feitas por meio do método cinético no ultravioleta, considerando os valores de 26 a $174 \mathrm{UI} / \mathrm{L}$ como normais. Das 6.230 dosagens, 3.369 tinham também dosagens de FT4 (valor de referência 0,7 a $1,8 \mathrm{ng} / \mathrm{dL}$ ).

$\mathrm{Na}$ análise do grupo estudado, foi avaliada a correlação entre CPK e TSH. Os dados são mostrados como mediana (intervalo) por ser uma distribuição não Gaussiana e sua correlação foi calculada pelo teste de Spearman. Categorizaram-se as dosagens realizadas em hipotireoidismo ( $\mathrm{TSH}>4,0 \mathrm{mU} / \mathrm{L}$ ) e hipertireoidismo ( $\mathrm{TSH}<0,4 \mathrm{mU} / \mathrm{L}$ ), comparando com o grupo controle - eutireoidismo (TSH entre 0,4 e 4,0 $\mathrm{mU} / \mathrm{L})$. Dividiu-se, ainda, o grupo que tinha dosagem de FT4 disponível em hipertireoidismo (TSH $<0,4$ $\mathrm{mU} / \mathrm{L}$ e FT4 > 1,8 ng/dL), hipertireoidismo subclínico ( TSH $<0,4 \mathrm{mU} / \mathrm{L}$ e FT4 entre 0,7 e $1,8 \mathrm{ng} / \mathrm{dL}$ ), eutireoidismo (TSH entre 0,4 e 4,0 mU/L e FT4 entre 0,7 e 1,8 ng/dL), hipotireoidismo subclínico (TSH $>4,0$ $\mathrm{mU} / \mathrm{L}$ e FT4 entre 0,7 e $1,8 \mathrm{ng} / \mathrm{dL}$ ) e hipotireoidismo $(\mathrm{TSH}>4,0 \mathrm{mU} / \mathrm{L}$ e FT4 $<0,7 \mathrm{ng} / \mathrm{dL})$. Analisou-se a distribuição da CPK entre os grupos mediante comparação múltipla de Newman-Keuls, e a diferença da variância de cada grupo foi identificada pelo ANOVA. Um $\mathrm{p}$ menor que 0,05 foi considerado para indicar significância estatística e intervalos de confiança 95\% (IC95) foram calculados. Os dados, quando não paramétricos, são mostrados como mediana (intervalo). As análises estatísticas foram realizadas pelo GraphPad Prism 4.0.

\section{RESULTADOS}

A mediana do TSH nos exames laboratoriais avaliados foi de 1,62 mU/L (0,01-75,00), e a de CPK foi 108 $\mathrm{U} / \mathrm{L}$ (17-4.688). A correlação entre os valores de TSH e de CPK, medida pelo coeficiente de Spearman, apesar de estatisticamente significante, foi muito fraca $(\mathrm{r}=$ 0,065 [IC95\% 0,040 a 0,09l], p < 0,0001). Considerando apenas o grupo que tinha dosagem de FT4, tal correlação foi semelhante $(r=0,083$ [IC95\% 0,048 a $0,12], \mathrm{p}<0,0001)$, enquanto a correlação entre CPK e FT4 foi inversamente proporcional, embora também fraca $(r=-0,091[$ IC95\% $-0,13$ a $-0,056], p<0,0001)$.

Quando se estratificou o grupo inteiro $(\mathrm{N}=6.230)$ de acordo com a função tireoidiana (hipertireoidismo, eutireoidismo, hipotireoidismo), encontraram-se 586 $(9,4 \%)$ dosagens compatíveis com hipertireoidismo, 556 $(8,9 \%)$ em níveis de hipotireoidismo e 5.088 (81,7\%), eutireoidianos. O estado da função tireoidiana no momento da coleta não indica, necessariamente, o diagnóstico do paciente. Pacientes com diagnóstico de hipotireoidismo poderiam, momentaneamente, estar em hipertireoidismo por uma dose excessiva de reposição da levotiroxina e, para o estudo, foram classificados no grupo de hipertireoidismo. Ao contrário, pacientes em hipertireoidismo com dose excessiva de droga antitireodiana e TSH elevado foram classificados no grupo de hipotireoidismo.

No grupo do hipertireoidismo, a mediana de TSH foi de $0,16 \mathrm{mU} / \mathrm{L}(0,01-0,39)$ e de CPK, $98 \mathrm{U} / \mathrm{L}$ (27-1.113). Entre as amostras classificadas como hipotireoidismo, os valores de TSH foram $5,24 \mathrm{mU} / \mathrm{L}$ (4,01-75,00) e CPK 114 U/L (25-4.182), enquanto naqueles com eutireoidismo a mediana de TSH foi 1,63 $\mathrm{mU} / \mathrm{L}(0,40-4,00)$ e CPK $108 \mathrm{U} / \mathrm{L}$ (17-4688). As diferenças entre o TSH e CPK de todos os grupos foram estatisticamente significantes ( $\mathrm{p}<0,01)$ (Tabela 1 ).

Avaliando então o subgrupo que tinha também dosagem de FT4 disponível, observou-se que, à medida que o TSH aumentava, a CPK também aumentava, sendo menor no grupo com hipertireoidismo clínico e maior no grupo de hipotireoidismo clínico (Tabela 2). 
Tabela 1. Valores mínimos, máximos e das medianas do CPK e do TSH de cada um dos grupos de exames, classificados conforme os estados patológicos tireoidianos e níveis hormonais normais

\begin{tabular}{lcccc}
\hline & $\begin{array}{c}\text { Hipertireoidismo } \\
(\mathbf{n = 5 8 6 )}\end{array}$ & $\begin{array}{c}\text { Eutireoidismo } \\
(\mathbf{n = 5 . 0 8 8 )}\end{array}$ & $\begin{array}{c}\text { Hipotireoidismo } \\
(\mathbf{n = 5 5 6 )}\end{array}$ & $\mathbf{p}$ \\
\hline CPK (U/L) mediana (mín-máx) & $98(27-1.113)$ & $108(17-13.904)$ & $114(25-4.182)$ & $<0,01$ \\
TSH (mU/L) mediana (mín-máx) & $0,16(0,01-0,39)$ & $1,63(0,40-4,00)$ & $5,24(4,01-75,00)$ & $<0,01$ \\
\hline
\end{tabular}

Tabela 2. Valores mínimos, máximos e das medianas do CPK, TSH e T4 livre dos grupos com níveis hormonais clínicos e subclínico e o grupo em eutireoidismo no momento do exame

\begin{tabular}{|c|c|c|c|c|c|c|}
\hline & $\begin{array}{l}\text { Hipertireoidismo } \\
(\mathrm{n}=108)\end{array}$ & $\begin{array}{c}\text { Hipertireoidismo } \\
\text { subclínico }(n=299)\end{array}$ & $\begin{array}{l}\text { Eutireoidismo } \\
(\mathrm{n}=2.633)\end{array}$ & $\begin{array}{c}\text { Hipotireoidismo } \\
\text { subclínico }(n=313)\end{array}$ & $\begin{array}{l}\text { Hipotireoidismo } \\
\quad(n=16)\end{array}$ & $\mathbf{p}$ \\
\hline CPK (U/L) & $86(32-610)$ & 99 (29-925) & 105 (17-4.688) & $111(32-1.064)$ & $170(60-947)$ & $<0,001$ \\
\hline TSH (mU/L) & $0,06(0,01-0,39)$ & $0,19(0,01-0,39)$ & $1,60(0,40-4,00)$ & $5,24(4,01-15,80)$ & $29,80(8,23-75,00)$ & $<0,001$ \\
\hline T4 livre (ng/dL) & $2,03(1,81-3,45)$ & $1,44(0,51-1,80)$ & $1,23(0,51-2,36)$ & $1,14(0,71-1,80)$ & $0,58(0,30-0,68)$ & $<0,001$ \\
\hline
\end{tabular}

\section{DISCUSSÃO}

Foi demonstrado neste estudo que a mediana da enzima CPK sérica dos pacientes avaliados encontra-se dentro dos parâmetros estabelecidos, isso porque provavelmente a maioria dos pacientes realizou os exames sem queixas musculares específicas, mas apenas para monitorizar um tratamento realizado, por exemplo, com estatinas. A CPK correlacionou-se fraca e positivamente com o TSH $(\mathrm{r}=0,065)$ e negativamente com o FT4 $(\mathrm{r}=-0,083)$. Ao se estratificar pelo status tireoidiano, observaram-se valores de CPK mais elevados no grupo de hipotireoidismo e menores no grupo de hipertireoidismo, sendo essas diferenças estatisticamente significantes (Tabela 1). Ratificando esse resultado, a análise do grupo com o FT4 mostrou que a CPK na apresentação subclínica do hipotireoidismo foi maior que a do grupo normal, porém menor que no grupo de hipotireoidismo clínico. No grupo de hipertireoidismo subclínico, a CPK foi menor que no normal, porém maior que do hipertireoidismo clínico.

Quanto ao mecanismo do hipertireoidismo relacionado à miopatia, ocorreria um desacoplamento da fosforilação pela quinase. Esse processo poderia se dar ao nível mitocondrial em que, pelo estado de hipermetabolismo, ocorreria o aumento da produção da isoforma neonatal da creatina quinase, pouco efetiva, em detrimento da enzima mitocondrial. Com isso, haveria diminuição da CPK muscular (9). Apesar de aumentar excessivamente os canais de cálcio, não haveria aumento na eficiência muscular contrátil, pela diminuição do gradiente de concentração da membrana, traduzindo-se clinicamente como fraqueza. Somado a isso, o estado de hipermetabolismo poderia aumentar a depleção proteica e diminuir a síntese da CPK (10).
O próprio hormônio tireoidiano está relacionado à regulação da proliferação e maturação de miócitos (11). Quando em excesso, aumenta o tempo da mitose do músculo estriado e o intervalo necessário para a divisão celular. Por isso, no hipertireoidismo pode haver consequente redução da massa muscular e da quantidade de CPK (12).

Por outro lado, apesar das explicações quanto a seu mecanismo fisiopatológico, há divergências quanto à relação do hipertireoidismo e a alteração na CPK. Alguns estudos não mostram diferença de enzimas musculares séricas entre hipertireoidismo e eutireoidismo (13). No entanto, outras evidências mostram pacientes com hipertireoidismo e diminuição de várias enzimas relacionadas ao equilíbrio hidroeletrolítico (14), como também diminuição de enzimas musculares em comparação com indivíduos com níveis normais de hormônios tireoidianos, sendo esses fatores relacionados à diminuição da contratilidade muscular $(15,16)$. Adicionalmente, a carnitina, que está envolvida no metabolismo energético celular, mostra ter a mesma tendência da CPK em relação aos hormônios tireoidianos que encontramos em nosso estudo (6). A variação dos níveis de enzimas musculares retorna ao patamar de normalidade com o tratamento do hipertireoidismo (15).

O hipotireoidismo é uma causa bem estabelecida de rabdomiólise (17), e mesmo sua apresentação subclínica tem a possibilidade de afetar os músculos esqueléticos (18). Pacientes com hipertireoidismo tratados com drogas antitireoidianas, radioiodoterapia ou tireoidectomias podem também apresentar rabdomiólise $(19,20)$, sugerindo que um hipotireoidismo relativo possa também levar a essa lesão muscular (21). 
A apresentação da doença muscular pode ser atípica, podendo ter níveis muito elevados de enzimas musculares sem apresentar fraqueza muscular clinicamente evidente (22). Com o tratamento do hipotireoidismo com levotiroxina, geralmente há reversão dos marcadores de lesão muscular (23), muitas vezes até mais rapidamente que a normalização do TSH (24).

Um mecanismo sugerido para explicar a elevação da CPK sérica pelo hipotireoidismo é que a diminuição do hormônio tireoidiano causasse uma redução no clearance da CPK. Essa hipótese foi criticada, pois outros trabalhos já mostram que outras enzimas musculares, como a troponina, não se relacionam com a função tireoidiana (25).

Alternativamente, a maioria dos estudos aponta para uma redução da capacidade funcional celular a partir da diminuição dos canais de cálcio ATP dependentes do retículo sarcoplasmático como possível explicação para essas alterações (26). Esse mecanismo seria possivelmente mediado pelo gene PGC-l $\alpha$, que sofreria influência dos hormônios tireoidianos (27). Como uma tentativa de regulação, haveria alteração da quantidade da $\mathrm{CPK}$, embora não melhore a funcionalidade celular, pois $50 \%$ da atividade dos canais de cálcio dependem de outras enzimas que são limitantes para o processo (28). Com isso, mesmo não tendo influência direta na atividade da CPK, a disfunção do canal de cálcio induziria um aumento de CPK compensatória. Além disso, a própria membrana mitocondrial, em situações de hipotireoidismo, sofreria alterações nas moléculas de cardiolipina, prejudicando o mecanismo fisiológico de acoplamento da fosforilação oxidativa mitocondrial (29).

Essa correlação entre TSH e CPK tem importância fundamental na condução do paciente com distúrbios tireoidianos, principalmente no caso do hipotireoidismo. Sabemos, por exemplo, que o hipotireoidismo é uma causa secundária de hipercolesterolemia e que, com a normalização da função tireoidiana, a colesterolemia usualmente normaliza. Porém, alguns pacientes são tratados com estatinas, aumentando ainda mais a possibilidade de elevação da CPK. Outras medicações que podem causar lesões musculares (antibióticos macrolídios, antifúngicos azólicos, ciclosporina, e fibratos) (30) podem também ser utilizadas por pacientes com hipotireoidismo, aumentando mais ainda a chance de elevação de CPK nesses pacientes já predispostos a tal.

O trabalho tem a limitação de ser um estudo transversal retrospectivo, havendo necessidade de se investigar fatores externos, como doenças ou uso de me- dicações, que possam interferir na dosagem do CPK. Entretanto, acreditamos que possíveis vieses possam ser diluídos pela grande amostra arrolada. Embora possa ter havido algum desvio de seleção, pois se coletaram os dados de exames solicitados a pacientes que tinham alguma indicação clínica, acreditamos que esse desvio não deve ter interferido nos resultados. Devemos atentar ainda para o fato de que, pela forma de seleção dos pacientes, a população estudada não pode servir como base para referência da prevalência das patologias tireoidianas em nossa região.

Concluímos que existe uma correlação positiva entre CPK sérica e TSH e negativa entre CPK e T4 livre, sendo essa progressivamente maior de acordo com os estados de hipertireoidismo, eutireoidismo e hipotireoidismo, respectivamente, fortalecendo a evidência da relação entre a função tireoidiana e o metabolismo enzimático muscular.

Declaração: os autores declaram não haver conflitos de interesse científico neste estudo.

\section{REFERÊNCIAS}

1. Kimura N, Hamaoka T, Kurosawa Y, Katsumura T. Contribution of intramuscular oxidative metabolism to total ATP production during forearm isometric exercise at varying intensities. Tohoku J Exp Med. 2006;208(4):307-20.

2. Longo N, Ardon O, Vanzo R, Schwartz E, Pasquali M. Disorders of creatine transport and metabolism. Am J Med Genet C Semin Med Genet. 2011;157(1):72-8.

3. Korge P, Byrd SK, Campbell KB. Functional coupling between sarcoplasmic-reticulum-bound creatine kinase and $\mathrm{Ca}(2+)$-ATPase. Eur J Biochem. 1993;213(3):973-80.

4. Dillmann WH. Cellular action of thyroid hormone on the heart. Thyroid. 2002;12(6):447-52.

5. Suarez J, Scott BT, Suarez-Ramirez JA, Chavira CV, Dillmann WH. Thyroid hormone inhibits ERK phosphorylation in pressure overload-induced hypertrophied mouse hearts through a receptor-mediated mechanism. Am J Physiol Cell Physiol. 2010;299(6):C1524-9.

6. Sinclair C, Gilchrist JM, Hennessey JV, Kandula M. Muscle carnitine in hypo- and hyperthyroidism. Muscle Nerve. 2005;32(3):357-9.

7. Fleischman A, Makimura H, Stanley TL, McCarthy MA, Kron M, Sun $\mathrm{N}$, et al. Skeletal muscle phosphocreatine recovery after submaximal exercise in children and young and middle-aged adults. J Clin Endocrinol Metab. 2010;95(9):E69-74.

8. Duyff RF, Van den Bosch J, Laman DM, van Loon BJ, Linssen WH. Neuromuscular findings in thyroid dysfunction: a prospective clinical and electrodiagnostic study. J Neurol Neurosurg Psychiatry. 2000;68(6):750-5.

9. Kemp GJ, Manners DN, Clark JF, Bastin ME, Radda GK. Theoretical modelling of some spatial and temporal aspects of the mitochondrion/creatine kinase/myofibril system in muscle. Mol Cell Biochem. 1998;184(1-2):249-89.

10. Seppet EK, Saks VA. Thyroid hormones and the creatine kinase system in cardiac cells. Mol Cell Biochem. 1994;133-134:299-309.

11. Chattergoon NN, Giraud GD, Thornburg KL. Thyroid hormone inhibits proliferation of fetal cardiac myocytes in vitro. J Endocrinol. 2007;192(2):R1-8. 
12. Ledda-Columbano GM, Molotzu F, Pibiri M, Cossu C, Perra A, Columbano $A$. Thyroid hormone induces cyclin D1 nuclear translocation and DNA synthesis in adult rat cardiomyocytes. FASEB J. 2006;20(1):87-94.

13. Mula-Abed WA, Al-Sinani SS, Al-Hashmi HS. Serum myoglobin in patients with thyroid dysfunction. Sultan Qaboos Univ Med J. 2009;9(1):37-41.

14. Suzuki S, Chen H, Takahashi T, Niwa O. Effects of hyper- and hypothyroidism on carbonic anhydrase, Mg2(+)-dependent ATPase and $\mathrm{Mg} 2(+)$-dependent, $\mathrm{HCO} 3(-)$-stimulated ATPase activities of rat duodenal mucosa and kidney cortex. J Endocrinol. 1990;126(1):119-29.

15. Roti E, Bandini P, Robuschi G, Emanuele R, Bolognesi R, Ciarlini $\mathrm{E}$, et al. Serum concentrations of myoglobin, creatine kinase, lactate dehydrogenase and cardiac isoenzymes in euthyroid, hypothyroid and hyperthyroid subjects. Ric Clin Lab. 1980;10(4):609-17.

16. Kasai K. Serum myoglobin level in altered thyroid states. J Clin Endocrinol Metab. 1979;48(1):1-4.

17. Kuo HT, Jeng CY. Overt hypothyroidism with rhabdomyolysis and myopathy: a case report. Chin Med J. 2010;123(5):633-7.

18. Hekimsoy Z, Oktem IK. Serum creatine kinase levels in overt and subclinical hypothyroidism. Endocr Res. 2005;31(3):171-5.

19. Benavides VC, Rivkees SA. Myopathy associated with acute hypothyroidism following radioiodine therapy for graves disease in an adolescent. Int J Pediatr Endocrinol. 2010;2010:717303.

20. Shaheen D, Kim CS. Myositis associated with the decline of thyroid hormone levels in thyrotoxicosis: a syndrome? Thyroid. 2009;19(12):1413-7.

21. Mizuno H, SugiyamaY, NishiY, Ueda N, OhroY,Togari H. Elevation of serum creatine kinase in response to medical treatment of Graves' disease in children. Acta Paediatr. 2006;95(2):243-5.
22. George G. Hypothyroidism presenting as puzzling myalgias and cramps in 3 patients. J Clin Rheumatol. 2007;13(5):273-5.

23. Shimoda SI, Kasai K. [A clinical evaluation of the increased serum myoglobin: creatine phosphokinase and lactic dehydrogenase in patients with thyroid disorders (author's transl)]. Nippon Naibunpi Gakkai Zasshi. 1980;56(8):1096-106.

24. Martino E, Sardano G, Vaudagna G, Bambini G, Breccia M, Motz E, et al. Serum myoglobin in primary hypothyroidism and effect of L-thyroxine therapy. J Nucl Med. 1982;23(12):1088-92.

25. Ness-Abramof R, Nabriski DA, Shapiro MS, Tripto-Shkolnik L, Katz B, Weiss $E$, et al. Cardiac troponin $T$ is not increased in patients with hypothyroidism. Intern Med J. 2009;39(2):117-20.

26. Docherty I, Harrop JS, Hine KR, Hopton MR, Matthews HL, Taylor CJ. Myoglobin concentration, creatine kinase activity, and creatine kinase $B$ subunit concentrations in serum during thyroid disease. Clin Chem. 1984;30(1):42-5.

27. Athea Y, Garnier A, Fortin D, Bahi L, Veksler V, Ventura-Clapier R. Mitochondrial and energetic cardiac phenotype in hypothyroid rat. Relevance to heart failure. Pflugers Arch. 2007;455(3):431-42.

28. Joubert F, Mateo P, Gillet B, Beloeil JC, Mazet JL, Hoerter JA. CK flux or direct ATP transfer: versatility of energy transfer pathways evidenced by NMR in the perfused heart. Mol Cell Biochem. 2004;256-257(1-2):43-58.

29. Saks V, Kaambre T, Guzun R, Anmann T, Sikk P, Schlattner U, et al. The creatine kinase phosphotransfer network: thermodynamic and kinetic considerations, the impact of the mitochondrial outer membrane and modelling approaches. Subcell Biochem. 2007;46:27-65.

30. Sousa AA, Kronit HS, Neves Fde A, Amato AA. Fenofibrate-induced rhabdomyolysis in a patient with chronic kidney disease: an unusual presenting feature of hypothyroidism. Arq Bras Endocrinol Metabol. 2009;53(3):383-6. 